\title{
Probabilistic alternatives for competitive analysis
}

Citation for published version (APA):

Hiller, B., \& Vredeveld, T. (2009). Probabilistic alternatives for competitive analysis. METEOR, Maastricht University School of Business and Economics. METEOR Research Memorandum No. 053

https://doi.org/10.26481/umamet.2009053

Document status and date:

Published: 01/01/2009

DOI:

10.26481/umamet.2009053

Document Version:

Publisher's PDF, also known as Version of record

\section{Please check the document version of this publication:}

- A submitted manuscript is the version of the article upon submission and before peer-review. There can be important differences between the submitted version and the official published version of record.

People interested in the research are advised to contact the author for the final version of the publication, or visit the DOI to the publisher's website.

- The final author version and the galley proof are versions of the publication after peer review.

- The final published version features the final layout of the paper including the volume, issue and page numbers.

Link to publication

\footnotetext{
General rights rights.

- You may freely distribute the URL identifying the publication in the public portal. please follow below link for the End User Agreement:

www.umlib.nl/taverne-license

Take down policy

If you believe that this document breaches copyright please contact us at:

repository@maastrichtuniversity.nl

providing details and we will investigate your claim.
}

Copyright and moral rights for the publications made accessible in the public portal are retained by the authors and/or other copyright owners and it is a condition of accessing publications that users recognise and abide by the legal requirements associated with these

- Users may download and print one copy of any publication from the public portal for the purpose of private study or research.

- You may not further distribute the material or use it for any profit-making activity or commercial gain

If the publication is distributed under the terms of Article $25 \mathrm{fa}$ of the Dutch Copyright Act, indicated by the "Taverne" license above, 


\section{Maastricht University}

Benjamin Hiller, Tjark Vredeveld

Probabilistic alternatives for competitive analysis

RM/09/053

\section{METEOR}

Maastricht University School of Business and Economics

Maastricht Research School of Economics

of Technology and Organization

P.O. Box 616

NL - 6200 MD Maastricht

The Netherlands 


\title{
Probabilistic alternatives for competitive analysis
}

\author{
Benjamin Hiller* Tjark Vredeveld ${ }^{\dagger}$
}

November 5, 2009

\begin{abstract}
In the last 20 years competitive analysis has become the main tool for analyzing the quality of online algorithms. Despite of this, competitive analysis has also been criticized: it sometimes cannot discriminate between algorithms that exhibit significantly different empirical behavior or it even favors an algorithm that is worse from an empirical point of view. Therefore, there have been several approaches to circumvent these drawbacks. In this survey, we discuss probabilistic alternatives for competitive analysis.
\end{abstract}

\section{Introduction}

Classical optimization techniques assume complete knowledge of the data relevant to solve the optimization problem. However, this assumption is not always realistic, since quite often decisions need to be made without having access to all of the data. Such problems are called online optimization problems. The major feature of these problems is that the input is only revealed over time, while an online algorithm has to take decisions that cannot be revoked once more information becomes known. The following three problems are classical examples.

Paging This problem models an optimization problem occuring in a two-level memory system. The slow memory stores a fixed set $M$ of pages. To speed up access to the pages, up to $k$ pages can be put in the fast memory or cache. The task is to serve a sequence $\sigma \in M^{n}$ of $n$ page requests. In order to serve a page request $p \in M$, the page has to be in the cache, otherwise a page fault occurs. The requested page must then be loaded into the cache, and, if the cache contains $k$ pages, at least one page must be evicted from the cache. An online paging algorithm needs to decide which page(s) will be evicted from the cache on a page fault without knowing the remaining request sequence or its length. The goal is to minimize the number of page faults.

\footnotetext{
*Zuse Institute Berlin, Takustraße 7, D-14195 Berlin, Germany, hiller@zib.de

${ }^{\dagger}$ Maastricht University, Department of Quantitative Economics, P.O.Box 616, 6200 MD, Maastricht, The Netherlands, t.vredeveld@maastrichtuniversity.nl
} 
Bin Packing There is an (unlimited) number of unit capacity bins and a sequence of items, described by their item sizes $s_{1}, \ldots, s_{n}$. The task is to pack each item into a bin that still has sufficient capacity. Once an item has been packed in one bin, it is not possible to repack it into another bin. An online algorithm has to determine the bin for each item without knowing the number of items to come and their sizes. The goal is to minimize the total number of bins used.

Scheduling In machine scheduling, there are $n$ jobs, each of which needs to be processed on one of $m$ machines. A machine can process at most one job at a time and each job can be processed by at most one machine at a time. Moreover, it cannot be processed before its release date. Processing a job $j$ takes $p_{j}$ amounts of time and we consider both a model in which the processing may be interrupted (preemptive model) and one in which a job needs to be processed until completion once started (non-preemptive model). The goal is to schedule the jobs on the machines in such a way that a certain objective function is minimized. In this paper, we consider the total weighted completion time and the total flow-time. The completion time of a job is the earliest point in time at which a job has received $p_{j}$ units of processing time, whereas the flow-time is the time a job is in the system, i.e., its completion time minus its release date. The total weighted completion time is the sum over all jobs of its weight, $w_{j}$, times its completion time, whereas the total flow-time is, of course, the sum of all flow-times. In scheduling, there are two types of uncertainty. First, the uncertainty about jobs to come (real online) and secondly, whenever there is knowledge on the existence of the jobs, the processing times can be unknown.

When designing an online algorithm, one faces the question of how to measure the quality of this algorithm. By far the most often used measure is competitive analysis [43], in which an online algorithm is compared to an optimal offline algorithm that knows the entire input in advance. In some cases, competitive analysis does not provide satisfying answers, e. g., it sometimes cannot discriminate between algorithms that exhibit significantly different empirical performance. The approach of competitive analysis and some of the criticism voiced against it are discussed in Section 2.

There are several different approaches to circumvent these drawbacks. In this survey, we focus on probabilistic approaches to analyze online algorithms to obtain more detailed results than possible with competitive analysis. Section 3 gives an overview on these approaches and shows how they help to get improved results for the three problems introduced above.

\section{Competitive analysis and its variants}

The standard theoretical tool used to assess online algorithms is competitive analysis, which is widely used. Probably the first time competitive analysis has been performed was in 1966 by Graham [21]. However, he did not mention the name competitive analysis nor online optimization. Only with the seminal paper of Sleator and Tarjan [43] competitive analysis became the standard yardstick. The term competitive analysis was first proposed by Karlin et al. [28]. 
The basic idea is to evaluate the "loss" in solution quality due to lack of information in the worst case. Competitive analysis compares the performance of a given online algorithm ALG to that of an algorithm that knows the complete request sequence in advance and can serve the requests at minimum cost. This benchmark algorithm is called optimal offline algorithm OPT. For a request sequence $\sigma$, we denote the corresponding optimal offline cost and the cost of ALG by $\operatorname{OPT}(\sigma)$ and $\operatorname{ALG}(\sigma)$, respectively. The algorithm ALG is said to be $c$-competitive for $c \geq 1$ and some $b \geq 0$, if

$$
\operatorname{ALG}(\sigma) \leq c \cdot \mathrm{OPT}(\sigma)+b
$$

for all request sequences $\sigma$. The competitive ratio of ALG is the smallest value $c$ such that ALG is c-competitive. ALG is called competitive if ALG is ccompetitive for some constant $c \geq 1$ that does not depend on the length of the sequence. Frequently, only the case $b=0$ is considered, which makes the results less asymptotic.

Although there are many cases where competitive analysis can be applied successfully, i. e., there are $c$-competitive online algorithms for some small $c$, competitive analysis fails to give useful results for other important problems. The main reason is that the results are too pessimistic, since an online algorithm needs to safeguard against any possible future. We will highlight these issues for our three example problems.

Paging Well-known paging algorithms are flush-when-full (FWF), which evicts every page on a page fault, first-in-first-out (FIFO), which evicts the page that has been in the cache the longest, and least-recently-used (LRU), which evicts the page whose most recent request was earliest.

Sleator and Tarjan [43] showed that the competitive ratio of every online algorithm is at least $k$. They also proved that both LRU and FIFO attain this ratio and are thus optimal, whereas some other algorithms are not $k$-competitive. Later it was shown that any online algorithm that is a marking algorithm is $k$-competitive [46], which includes FWF and LRU.

These results did not match empirical observations: LRU was observed to be much better than FWF and to outperform FIFO. Moreover, the "empirical" competitive ratio of LRU was much smaller than $k$.

Bin Packing Probably the simplest online algorithm for bin packing is NextFit, which looks only at the most recently opened bin. If the next item fits, it is put into this bin; if not, the item is put in a new bin and the other bin is never considered again. It is shown by Johnson [25] that the competitive ratio of NextFit is 2 . The algorithm FirstFit scans through the bins in their opening order, putting the item in the first bin with sufficient capacity. If no bin is found, a new bin is opened to accomodate the item. BestFit works similar, but puts the item in the open bin with least remaining capacity that suffices for the item. Both FirstFit and BestFit have competitive ratio 1.7 [26], but BestFit gives better results in practice. Yao [49] proposes RefinedFirstFit, which achieves a competitive ratio of $5 / 3$ based on a classification of items and bins according to sizes / remaining capacity. The Harmonic algorithm by Lee and Lee [33] classifies items by size and puts different items into the same bin if and 
only if they belong to the same class. Successively, this idea was pushed further and the best known algorithm so far achieves competitive ratio not more than 1.589 [38]. Van Vliet [47] proves a lower bound of 1.540 for the competitive ratio of any online algorithm.

Scheduling On a single machine, the problem of minimizing the flow-time in the preemptive model can be solved optimally by an online algorithm known as Shortest Remaining Processing Time [40]. This algorithm processes at any time the unfinished job that has smallest remaining processing time. On the other hand, in non-clairvoyant scheduling, i.e., nothing is known about a job's processing time until the job is completed, it can be shown that any deterministic online algorithm has a competitive ratio of $\Omega\left(n^{1 / 3}\right)$ or $\Omega(P)$ [36], where $n$ is the number of jobs and $P$ is the ratio between the maximum and minimum processing time. For the nonpreemptive problem on a single machine to minimize the total weighted completion time, there exists a 2-competitive online algorithm [2], which is the best possible [24]. For multiple machines, the best known online algorithm achieves a competitive ratio of 2.62 [18], whereas no online algorithm can be better than 1.309-competitive [48]. For an overview on other results in online scheduling, we refer to [37].

Competitive analysis of online algorithms can be viewed as a two player game. The online player, i. e., the online algorithm, tries to achieve a small competitive ratio, whereas the other player, the adversary, tries to generate the request sequence such that he can serve it with small cost, but incurring high cost for the online player, which gives a large competitive ratio.

Basically there are three approaches to remedy the drawbacks of competitive analysis in order to get more realistic and useful results.

1. Keep the idea of comparing to the offline optimum, but reduce the power of the adversary online algorithms are compared to.

2. Compare online algorithms to a weaker optimum, and use this to compare the algorithms.

3. Compare online algorithms directly instead of indirectly via the offline optimum.

The first approach is the most prominent one. An obvious way to reduce the power of the adversary is to restrict the set of request sequences it may generate. This problem-specific technique is quite successful. Examples for paging are the restriction to sequences that exhibit locality of reference $[1,46]$ or that are derived from an access graph [11]. Another possibility is to allow the online algorithm to use more resources (e.g., faster machines) than the adversary [27]; this is known as resource augmentation. Many more deterministic approaches have been proposed, but we will now focus on probabilistic ones.

\section{Alternative probabilistic measures}

Randomness can be used in two ways in the analysis of online algorithms. First, the request sequence may be assumed to be drawn from some probability distribution. This is equivalent to replacing the adversary by some random process. 
Second, the online algorithms themselves may be randomized, which makes it harder for the adversary to come up with bad sequences.

\subsection{Randomized Online Algorithms}

Using randomization to improve online algorithms has been suggested by Borodin et al. [12]. Usually, analyses are done with respect to an oblivious adversary, i. e., this adversary knows the probability distribution employed by the randomized online algorithm, but not the random outcomes. The competitive ratio is then determined by replacing ALG by its expectation $\mathbb{E}[\mathrm{ALG}]$ in Equation (1).

For the paging problem, Fiat et al. [19] showed a lower bound of $H_{k}\left(H_{k}\right.$ is the $k$ th harmonic number) on the competitive ratio of every randomized paging algorithm. Moreover, they provided a randomized paging algorithm that is $2 H_{k}$-competitive against the oblivious adversary. McGeoch and Sleator [34] presented an optimal randomized algorithm with competitive ratio $H_{k}$. Since $H_{k} \in$ $\mathcal{O}(\log k)$, both results show that randomized paging algorithms can achieve a significantly better competitive ratio than deterministic ones. Similar improvements have been achieved for many online optimization problems.

Interestingly, randomized algorithms for bin packing can only be slightly better than deterministic ones. Chandra [13] proves a lower bound of 1.536 for the competitive ratio of any randomized online algorithm, whereas the bestknown deterministic algorithms achieves 1.589 .

For the non-clairvoyant single machine scheduling problem to minimize the total flow-time, Becchetti and Leonardi [7] proposed a randomized online algorithm with competitive ratio of $\mathcal{O}(\log n)$ matching the lower bound of [36]. For the nonpreemptive problem on multiple machines to minimize the total weighted completion time, no randomized online algorithm can be better than 1.157 [41], whereas Correa and Wagner provide a randomized online algorithm with competitive ratio strictly less than 2 [18].

\subsection{Average case analysis}

Probably the first alternative for worst case analysis that comes into ones mind is average case analysis. In average case analysis, the request sequence is chosen according to some probability distribution. In most average case competitive analyses, the requests are chosen independently and identically distributed. In this context, the optimal value for a request sequence of length $n, \mathrm{OPT}_{n}$, becomes a random variable, just as $\mathrm{ALG}_{n}$, the value obtained by an (online) algorithm. The expected competitive ratio is defined as

$$
R_{\mathrm{ALG}}(n)=\mathbb{E}\left[\frac{\mathrm{ALG}_{n}}{\mathrm{OPT}_{n}}\right] .
$$

An alternative measure for the average case performance, would be the ratio of the expectations, i.e., the ratio $\mathbb{E}\left[\mathrm{ALG}_{n}\right] / \mathbb{E}\left[\mathrm{OPT}_{n}\right]$. This is the performance measure that is common to evaluate approximative stochastic scheduling policies, see, e.g., [35]. However, the notion of the optimum is different for stochastic scheduling and competitive analysis. In stochastic scheduling, one asks the question "How good can we do given the uncertainty that we encounter?" That is, the optimal policy also underlies the uncertainty about the data and therefore, it is defined as the policy that optimizes the expected value of the solution. 
On the other hand, in competitive analysis, one asks the question "What is the cost of not knowing the future?" Therefore, in competitive analysis, the solution of an online algorithm is compared to the optimal solution value per instance and the optimum is defined as the solution obtained by an optimal algorithm that knows the instance a priori. Scharbrodt et al. [39] and Souza and Steger [44] elaborate on the difference between the two measures. Although the ratio of the expectations is normally easier to compute, they prefer the expected competitive ratio, for several reasons. First, they say that the ratio of expectations favours algorithms that perform well on instances for which the optimal solution value is large, due to the fact that instances with small solution value contribute little to the expected value of an algorithm. On the other hand, the expectation of the ratio favours algorithms that perform well on many instances. Furthermore, by Markov's inequality, one can easily derive bounds on the probability that for a randomly generated instance the ratio $\mathrm{ALG}_{n} / \mathrm{OPT}_{n}$ is more than a certain factor away from the expected competitive ratio.

There is a vast literature on average case analysis of bin packing algorithms (see e.g., [15, 14] for surveys). Many results for bin packing focus on the asymptotic expected competitive ratio of ALG, i.e.,

$$
R_{\mathrm{ALG}}^{\infty}(F):=\lim _{n \rightarrow \infty} R_{\mathrm{ALG}}(n),
$$

assuming that the input sequence is generated by choosing each item size i.i.d. from distribution $F$. In most cases, $F$ is a uniform distribution, e.g., $U[0,1]$, the uniform distribution on $[0,1]$. The first precise average case asymptotic analysis is by Coffman et al. [17] for the Next Fit algorithm. They showed that the asymptotic expected competitive ratio for Next Fit is $R_{\mathrm{NF}}^{\infty}(U[0,1])=4 / 3$. Lee and Lee [33] proved that their Harmonic algorithm achieves $R_{\mathrm{H}}^{\infty}(U[0,1])<$ 1.306. Bentley et al. [10] were able to show, somewhat suprisingly, $R_{\mathrm{FF}}^{\infty}(U[0,1])=$ 1, i. e., that FirstFit is asymptotically optimal. The result was surprising since the empirical value is significantly larger than 1 in simulations, even for large numbers of items. This motivated the study of the expected waste after $n$ items, i. e., the expected difference between the number of bins used by ALG and the total size of $n$ items. Analyzing the waste, Shor [42] was able to separate the performance of FirstFit and BestFit: While the expected waste of FirstFit grows as $\Omega\left(n^{2 / 3}\right)$, BestFit has expected waste $\Theta\left(\sqrt{n} \log ^{3 / 4} n\right)$. Both FirstFit and BestFit are thus asymptotically optimal, but the performance of BestFit converges faster.

In the area of scheduling, the expected competitive ratio has been studied by Coffman and Gilbert in 1985 [16], which appears to be the first result on non-asymptotic expected competitive ratio. They consider list scheduling algorithms for minimizing the makespan on identical machines, when processing times are independent, identically distributed from the uniform or exponential distribution. Scharbrodt et al. [39] consider the scheduling problem on identical machines so as to minimize the total completion time. They perform an expected competitive ratio analysis for the SEPT list scheduling algorithm, that schedules jobs according to nondecreasing expected processing time. Note that SEPT is only a semi-online algorithm as the jobs need to arrive in a specified order. They show that SEPT has a constant expected competitive ratio, whenever the processing times are exponentially distributed (not necessarily i.i.d.). It should be noted that in the stochastic scheduling setting in which the opti- 
mal policy is one that minimizes the expected total completion time, the SEPT rule is optimal for exponentially distributed processing times. Their analysis extends to a more general class of distributions. In the deterministic cae, if the jobs are ordered in non-decreasing realized processing times, then Kawaguchi and Kyan [30] showed that the worst case performance ratio is bounded by $(1+\sqrt{2}) / 2$. This worst case bound actually holds also for the weighted version of the problem, whenever the jobs are ordered in nondecreasing realized processing time over weight. Souza and Steger [44] extend the result of Scharbrodt et al. to the weighted version and show that the WSEPT rule admits a constant expected competitive ratio whenever the jobs are identically distributed, satisfying some additional probabilistic property.

\subsection{Diffuse adversaries}

As competitive analysis is often criticized for being too pessimistic due to its worst case character, average case analysis is often considered to be too optimistic. Moreover, in many cases the probability distributions analyzed are quite special and/or realistic probabilistic models cannot be analyzed or are not available.

In order to address these issues and to improve upon competitive analysis, Koutsoupias and Papadimitriou [31] proposed the diffuse adversary model. In this model, the offline adversary is replaced by the diffuse adversary, which chooses a probability distribution $D$ out of a family of distributions $\Delta$. The class $\Delta$ of distributions may be used to express some structural property of the inputs without sticking to a certain distribution. An algorithm ALG is called $c$ competitive against class $\Delta$ of request sequence distributions, if there is a $b \geq 0$ such that

$$
\mathbb{E}_{D}[\operatorname{ALG}(\sigma)] \leq c \cdot \mathbb{E}_{D}[\mathrm{OPT}(\sigma)]+b
$$

for all $D \in \Delta$, where the request sequence $\sigma$ is drawn according to $D$. Note that this definition generalizes both competitive analysis and average case analysis for the ratio of expectations.

To apply this approach to the paging problem, Koutsoupias and Papadimitriou propose a class of distributions $\Delta_{\epsilon}$. This class contains all distributions $D$ such that the conditional probability $D[p \mid \sigma]$ of page $p$ being requested after request sequence $\sigma$ satisfies $D[p \mid \sigma] \leq \epsilon$ for all $p$ and $\sigma$. Clearly, a small $\epsilon$ limits the power of the adversary since he has less control over the next request (note that $\Delta_{1}$ is equivalent to all request distributions). Koutsoupias and Papadimitriou show that LRU attains the optimal competitive ratio against $\Delta_{\epsilon}$ for any $\epsilon \in[0,1]$. However, they cannot determine this ratio. In subsequent work, Young [50] gives lower and upper bounds for the optimal competitive ratio in terms of a function $\psi(\epsilon, k)$ that match up to a factor of two. The function $\psi(\epsilon, k)$ exhibits the following threshold behavior around $\epsilon=1 / k$ :

$$
\psi(\epsilon, k) \text { is } \begin{cases}\leq 1+\ln \frac{1}{\delta} & \epsilon=(1-\delta) / k, \\ \approx \ln k & \epsilon=1 / k, \\ \leq k \frac{\delta}{1+\delta} & \epsilon=(1+\delta) / k .\end{cases}
$$

Thus the optimal competitive ratio (that of LRU) is constant for small $\epsilon$ and almost $k$ for large $\epsilon$. This bound holds for randomized algorithms, too, except 
for the case $\epsilon \geq 1 / k$ where both the lower and upper bounds are $\mathcal{O}(\log k)$. Moreover, Young shows that FIFO and FWF have competitive ratio $k$ for $\epsilon \geq$ $1 / k$. Hence, these results generalize the standard competitive analysis results and those for randomized algorithms and are able to discriminate between FWF or FIFO and LRU.

Becchetti [6] proposes a different diffuse adversary for the paging problem. The class $\Delta$ comprises distributions $D$ whose conditional distributions $D[p \mid \sigma]$ favor pages $p$ that are more recent w.r.t. $\sigma$ in order to model the locality of reference often encountered in practice. He then shows that LRU achieves a constant competitive ratio against $\Delta$, whereas that of FWF is $\Omega(k)$ if locality of reference is high.

\subsection{Smoothed competitive analysis}

An alternative compromise between worst case and average case analysis is to consider smoothed inputs. The notion of smoothed complexity was introduced by Spielman and Teng [45] in an attempt to explain the success of algorithms that are known to work well in practice while having a poor worst case performance. It can be seen as an hybrid between worst case and average case complexity. The basic idea is to randomly perturb the initial input instances and to analyze the expected performance of the algorithm on the perturbed instances. Becchetti et al. [8] extended the idea of smoothed complexity to smoothed competitive ratio. Following the idea of Spielman and Teng, they smoothen the input instance according to some probability distribution $f$. Given an input instance $\bar{I}$, let $N(\bar{I})$ denote the set of instances that can be obtained by smoothing the input instance $I$ according to $f$. The smoothed competitive ratio is defined as

$$
\sup _{\bar{I}} \mathbb{E}_{I \stackrel{f}{\leftarrow} N(\bar{I})}\left[\frac{\operatorname{ALG}(I)}{\mathrm{OPT}(I)}\right],
$$

where the supremum is taken over all input instances $\bar{I}$ and the expectation is taken according to $f$ over all instances $I$ in $N(\bar{I})$.

The adversarial input instance may be smoothed according to different smoothing models. The first class of models are symmetric models, which smooth a value by adding a random value. The distribution that is used to draw this random value from has mean 0 and is symmetric around this mean. The length of the interval from which the random value is taken may or may not depend on the original value.

The partial bit randomization model, introduced by [5, 9], is particularly useful if the input instance consists of $K$-bit integers. In this model the input values are not smoothed symmetrically. Assuming that each $\bar{x}_{j}$ is a $K$-bit integer, we perturb each $\bar{x}_{j}$ by replacing the $k$ least significant bits, for $0 \leq k \leq$ $K$, with some random number. Let $f$ be a probablility distribution over the interval $\left[0,2^{k}-1\right]$. Then we define the smoothed value as

$$
x_{j}=2^{k}\left\lfloor\frac{\bar{x}_{j}}{2^{k}}\right\rfloor+\varepsilon, \quad \text { where } \varepsilon \rightarrow f .
$$

For $k=0$, the smoothed values are equal to the initial values, whereas for $k=K$ the smoothed values are independent identically distributed from $\left[0,2^{K}-1\right]$.

Becchetti et al. [8] considered the so-called multilevel feedback (MLF) algorithm for preemptive scheduling on a single machine so as to minimize the 
total flow-time. When all processing times are between 1 and $2^{K}$, any nonclairvoyant deterministic online algorithm has a competitive ratio of $\Omega\left(2^{K}\right)$ and $\Omega\left(n^{1 / 3}\right)$. Becchetti et al. show that when the processing times are smoothed according to the partial bit randomization model, using a probability distribution with standard deviation $\sigma$, the smoothed competitive ratio of MLF is $\mathcal{O}\left(\left(2^{k} / \sigma\right)^{3}+\left(2^{k} / \sigma\right)^{2} 2^{K-k}\right)$. Whenever $f$ is the uniform distribution over $\left[0,2^{k}-1\right]$, this simplifies to $\mathcal{O}\left(2^{K-k}\right)$. Becchetti et al. also show that for the other smoothing models MLF has a lower bound of $\Omega\left(2^{K}\right)$ on the smoothed competitive ratio.

\subsection{Other measures}

As mentioned before, one weakness of competitive analysis is the comparison to the offline optimum, which is due to the fact that in a deterministic setting there is no reasonable concept of "optimal online algorithm" that can serve as a yardstick. In contrast, such an optimal online algorithm can be defined if the input is generated by some random process.

This approach has been applied in the analysis of paging algorithms even before the advent of competitive analysis. Franaszek and Wagner [20] studied the paging problem with request sequences generated according to the independent reference model. In this model, each request is generated independently and identically from the same fixed page distribution. They consider the page fault rate, i.e., the (asymptotic) expected number of page faults per time unit, instead of the number of page faults as their performance measure for paging algorithms. It is shown that no paging algorithm from a certain class of algorithms including LRU and FIFO achieves a page fault rate that is at most a constant factor larger than the optimal one. However, it turns out that if LRU is allowed to use (slightly) larger cache than the optimal online algorithm, the ratio of the page fault rates becomes bounded, which is not true for FIFO.

Karlin et al. [29] generalize this approach by considering a request sequence generated by a fixed Markov chain, which is a probabilistic version of the access graph model. Using the theory of Markov decision processes, they are able to characterize the optimal online algorithm for the given Markov chain, which is a deterministic algorithm whose decisions depend only on the current request and the state of the cache. They show that there are Markov chains such that all marking algorithms exhibit a page fault rate that is $\Omega(k)$ times the optimal one. Surprisingly, this includes LRU, which performs well under deterministic locality of reference models. The authors describe a polynomial-time algorithm whose page fault rate is not more than a constant times the optimal one. In contrast to most paging algorithms, this algorithm is not independent of the input, but depends crucially on the Markov chain generating the request sequence.

All the measures discussed so far use a single number to evaluate the performance of an algorithm, e. g., the maximum or the average. It may be more descriptive to look at the distribution of the performance of an algorithm for various inputs to assess the algorithm.

This approach was successfully applied by Hiller and Vredeveld [23] to the analysis of bin coloring algorithms. For the bin coloring problem [32], the input consists of a sequence of unit-sized items, each of which has one of $C$ colors. These items need to be packed sequentially into one of $m$ initially empty bins 
of capacity $B$. As soon as a bin is full, i. e., has exactly $B$ items, it is replaced by an empty one. As for bin packing, repacking is not allowed. An online algorithm must decide upon the bin for each item without knowing future item colors. The goal is to minimize the maximum number of different colors in one bin, which is called colorfulness. A natural algorithm for this problem is the algorithm GREEDYFIT: it packs an item with an already present color in the bin with that color and otherwise chooses a bin which currently has the least number of different colors. Another simple algorithm, ONEBın, packs all items in the same bin. Krumke et al. [32] analyzed these algorithms, showing that the competitive ratio of the trivial algorithm ONEBIN is better than that of GreedyFit. However, GreedyFit outperforms OneBin significantly in simulations.

Hiller and Vredeveld studied the bin coloring problem with random input, where a color sequence is generated by choosing each color i.i.d. according to a fixed color distribution. To compare the performance of algorithms, they use the concept of stochastic dominance. A random variable $X$ is stochastically dominated by a random variable $Y$, written $X \leq_{\mathrm{st}} Y$, if

$$
\operatorname{Pr}[X \geq x] \leq \operatorname{Pr}[Y \geq x] \quad \text { for all } x \in \mathbb{R} .
$$

Note that a stochastic dominance relation is a statement about the distributions of the random variables. Hiller and Vredeveld show that, for every color distribution, the maximum colorfulness of GREEDYFIT after $n$ items is stochastically dominated by that of ONEBin.

This result is particularly interesting for the uniform color distribution, since then the color sequences are uniformly distributed, too. It is then possible to interpret the probabilistic result deterministically as a counting result. Stochastic dominance of the maximum colorfulness distribution then implies that GREEDYFit achieves a low colorfulness on more instances than ONEBIN. In fact, stochastic dominance in this special case is equivalent to a recent deterministic way to compare online algorithms, known as Bijective Analysis [3]. Let $S_{n}$ denote the sequences of length $n$ and consider two online algorithms $\mathrm{ALG}_{1}$ and $\mathrm{ALG}_{2} . \mathrm{ALG}_{1}$ is said to dominate $\mathrm{ALG}_{2}$ w.r.t. Bijective Analysis, if there is a bijective mapping $\phi: S_{n} \rightarrow S_{n}$ such that $\operatorname{ALG}_{1}(\sigma) \leq \mathrm{ALG}_{2}(\phi(\sigma))$ for any $\sigma \in S_{n}$.

Very recently, Angelopoulos and Schweitzer [4] applied this idea to paging and showed that LRU is the unique optimal algorithm w.r.t. to Bijective Analysis for a restricted class of sequences exhibiting locality of reference. For the more general analysis of stochastic dominance, Hiller and Vredeveld [22] show that LRU is optimal with respect to probability distributions that model locality of reference.

\section{References}

[1] S. Albers, L. M. Favrholdt, and O. Giel. On paging with locality of reference. J. Comput. System Sci., 70(2):145-175, 2005.

[2] E. J. Anderson and C. N. Potts. On-line scheduling of a single machine to minimize total weighted completion time. Mathematics of Operations Research, 29:686-697, 2004. 
[3] S. Angelopoulos, R. Dorrigiv, and A. López-Ortiz. On the separation and equivalence of paging strategies. In Proceedings of the 18th ACM-SIAM Symposium on Discrete Algorithms, pages 229-237, 2007.

[4] S. Angelopoulos and P. Schweitzer. Paging and list update under bijective analysis. In Proceedings of the 20th ACM-SIAM symposium on Discrete algorithms, 2009. To appear.

[5] C. Banderier, R. Beier, and K. Mehlhorn. Smoothed analysis of three combinatorial problems. In Proceedings of the 28th International Symposium on Mathematical Foundations of Computer Science, volume 2747 of Lecture Notes in Computer Science, pages 198-207. Springer, 2003.

[6] L. Becchetti. Modeling locality: A probabilistic analysis of LRU and FWF. In Proceedings of the 12th European Symp. on Algorithms (ESA), pages 98109, 2004.

[7] L. Becchetti and S. Leonardi. Nonclairvoyant scheduling to minimize the total flow time on single and parallel machines. J. ACM, 51:517-539, 2004.

[8] L. Becchetti, S. Leonardi, A. Marchetti-Spaccamela, G. Schäfer, and T. Vredeveld. Average case and smoothed competitive analysis for the multi-level feedback algorithm. Math. Oper. Res., 31(1):85-108, 2006.

[9] R. Beier, A. Czumaj, P. Krysta, and B. Vöcking. Computing equilibria for congestion games with (im)perfect information. In Proceedings of the 15th Annual ACM-SIAM Symposium on Discrete Algorithms, pages 739-748, 2004.

[10] J. L. Bentley, D. S. Johnson, F. T. Leighton, C. C. McGeoch, and L. A. McGeoch. Some unexpected expected behavior results for bin packing. In Proceedings of the 16th Annual ACM Symposium on Theory of Computing, pages 279-288, 1984.

[11] A. Borodin, S. Irani, P. Raghavan, and B. Schieber. Competitive paging with locality of reference. J. Comput. System Sci., 50(2):244-258, 1995.

[12] A. Borodin, N. Linial, and M. E. Saks. An optimal on-line algorithm for metrical task systems. J. ACM, 39(4):745-763, 1992.

[13] B. Chandra. Does randomization help in on-line bin packing? Inform. Process. Lett., 43(1):15-19, 1992.

[14] E. G. Coffman, Jr., C. Courcoubetis, M. R. Garey, D. S. Johnson, P. W. Shor, R. R. Weber, and M. Yannakakis. Perfect packing theorems and the average-case behavior of optimal and online bin packing. SIAM Rev., 44(1):95-108, 2002.

[15] E. G. Coffman, Jr., M. R. Garey, and D. S. Johnson. Approximation algorithms for bin packing: A survey. In D. S. Hochbaum, editor, Approximation Algorithms for NP-hard problems. PWS Publishing, 1997.

[16] E. G. Coffman, Jr. and E. N. Gilbert. On the expected relative performance of list scheduling. Operations Research, 33(3):548-561, 1985. 
[17] E. G. Coffman, Jr., K. So, M. Hofri, and A. C. Yao. A stochastic model of bin-packing. Information and Control, 44:105-115, 1980.

[18] J. Correa and M. Wagner. LP-based online scheduling: From single machine to parallel machines. Mathematical Programming. To appear.

[19] A. Fiat, R. M. Karp, M. Luby, L. A. McGeoch, D. D. Sleator, and N. E. Young. Competitive paging algorithms. Journal of Algorithms, 12:685-699, 1991.

[20] P. A. Franaszek and T. J. Wagner. Some distribution-free aspects of paging algorithm performance. J. ACM, 21(1):31-39, 1974.

[21] R. L. Graham. Bounds for certain multiprocessor anomalies. Bell System Technical Journal, 45:1563-1581, 1966.

[22] B. Hiller and T. Vredeveld. On the optimality of least recently used. ZIBReport 08-39, Zuse Institute Berlin, 2008.

[23] B. Hiller and T. Vredeveld. Probabilistic analysis of online bin coloring algorithms via stochastic comparison. In Proceedings of the 16th Annual European Symposium on Algorithms, volume 5193 of Lecture Notes in Computer Science, pages 528-539. Springer, 2008.

[24] H. Hoogeveen and A. P. A. Vestjens. Optimal on-line algorithms for single-machine scheduling. In W. H. Cunningham, S. T. McCormick, and M. Queyranne, editors, Proceedings of the Fifth Conference on Integer Programming and Combinatorial Optimization IPCO, volume 1084 of Lecture Notes in Computer Science, pages 404-414, Berlin, 1996. Springer.

[25] D. S. Johnson. Fast algorithms for bin packing. J. Comput. System Sci., 8(8):272-314, 1974.

[26] D. S. Johnson, A. Demers, J. D. Ullman, M. R. Garey, and R. L. Graham. Worst-case performance bounds for simple one-dimensional packing algorithms. SIAM J. Comput., 3(4):299-325, 1974.

[27] B. Kalyanasundaram and K. Pruhs. Speed is as powerful as clairvoyance. J. ACM, 47(4):617-643, 2000.

[28] A. R. Karlin, M. S. Manasse, L. Rudolph, and D. D. Sleator. Competitive snoopy caching. Algorithmica, 3:70-119, 1988.

[29] A. R. Karlin, S. J. Phillips, and P. Raghavan. Markov paging. SIAM J. Comput., 30(2):906-922, 2000.

[30] T. Kawaguchi and S. Kyan. Worst case bound of an $\operatorname{lrf}$ schedule for the mean weighted flow-time problem. SIAM J. Comput., 15:1119-1129, 1986.

[31] E. Koutsoupias and C. Papadimitriou. Beyond competitive analysis. In Proceedings of the 35th Annual IEEE Symposium on Foundations of Computer Science, pages 394-400, 1994. 
[32] S. O. Krumke, W. E. de Paepe, L. Stougie, and J. Rambau. Online bin coloring. In F. M. auf der Heide, editor, Proceedings of the 9th Annual European Symposium on Algorithms, volume 2161 of Lecture Notes in Computer Science, pages 74-84, 2001.

[33] C. C. Lee and D. T. Lee. A simple online bin-packing algorithm. J. ACM, $32(3): 562-572,1985$.

[34] L. A. McGeoch and D. D. Sleator. A strongly competitive randomized paging algorithm. Algorithmica, 6:816-825, 1991.

[35] R. H. Möhring, A. S. Schulz, and M. Uetz. Approximation in stochastic scheduling: the power of LP-based priority policies. Journal of the ACM, 46:924-942, 1999.

[36] R. Motwani, S. Phillips, and E. Torng. Non-clairvoyant scheduling. Theoretical Computer Science, 130:17-47, 1994.

[37] K. Pruhs, J. Sgall, and E. Torng. Online scheduling. In J. Leung, editor, Handbook of Scheduling: Algorithms, Models, and Performance Analysis. CRC Press, 2004.

[38] M. B. Richey. Improved bounds for harmonic-based bin packing algorithms. Discrete Appl. Math., 34(1-3):203-227, 1991.

[39] M. Scharbrodt, T. Schickinger, and A. Steger. A new average case analysis for completion time scheduling. J. ACM, pages 121-146, 2006.

[40] L. Schrage. A proof of the optimality of the shortest remaining processing time discipline. Operations Research, 16:687-690, 1968.

[41] S. Seiden. A guessing game and randomized online algorithms. In Proceedings of the 32nd ACM Symposium on Theory of Computing, pages 592-601, 2000 .

[42] P. W. Shor. The average-case analysis of some on-line algorithms for bin packing. Combinatorica, 6(2):179-200, 1986.

[43] D. D. Sleator and R. E. Tarjan. Amortized efficiency of list update and paging rules. Comm. ACM, 28(2):202-208, 1985.

[44] A. Souza and A. Steger. The expected competitive ratio for weighted completion time scheduling. Theory of Computing Systems, 39:121-136, 2006.

[45] D. A. Spielman and S. H. Teng. Smoothed analysis of algorithms: Why the simplex algorithm usually takes polynomial time. J. ACM, 51:385-463, 2004.

[46] E. Torng. A unified analysis of paging and caching. Algorithmica, 20(1):175-200, 1998.

[47] A. van Vliet. On the asymptotic worst case behavior of harmonic fit. $J$. Algorithms, 20(1):113-136, 1996.

[48] A. P. A. Vestjens. On-line Machine Scheduling. PhD thesis, Eindhoven University of Technology, Netherlands, 1997. 
[49] A. C. Yao. New algorithms for bin packing. J. ACM, 27(2):207-227, 1980.

[50] N. E. Young. On-line paging against adversarially biased random inputs. J. Algorithms, 37(1):218-235, 2000. 